Mathematical Modelling And Analysis

Volume 20 Number 6, November 2015, 715-736

http://dx.doi.org/10.3846/13926292.2015.1108936

(c) Vilnius Gediminas Technical University, 2015
Publisher: Taylor\&Francis and VGTU

http://www.tandfonline.com/TMMA

ISSN: $1392-6292$

eISSN: $1648-3510$

\title{
Maximum-Profit Inventory Model with Stock-Dependent Demand, Time-Dependent Holding Cost, and All-Units Quantity Discounts
}

\author{
Hesham Kamal Alfares ${ }^{a}$ \\ ${ }^{a}$ King Fahd University of Petroleum \& Minerals \\ 31261 Dhahran, Saudi Arabia \\ E-mail: alfares@kfupm.edu.sa
}

Received September 30, 2014; revised September 27, 2015; published online November 15, 2015

\begin{abstract}
A new production-planning model with a unique set of realistic features is considered. First, the demand rate is a function of the current inventory level. Second, a new order is gradually produced according to a finite production rate. Third, the unit holding cost per time period is a function of both the unit purchase cost and the storage time duration. Fourth, the unit purchase cost is a function of the production lot size. Fifth, the starting/ending inventory for each cycle is a decision variable to be optimized. Finally, the objective of the model is to maximize the total profit per unit time. The purchase cost per unit decreases with larger lot size according to all-units quantity discount. On the other hand, the holding cost per unit increases with longer storage duration, either retroactively or incrementally. Mathematical models are formulated to represent this production planning system, and optimum solution procedures are developed.
\end{abstract}

Keywords: production planning, stock-dependent demand, variable holding cost, optimization, quantity discounts.

AMS Subject Classification: 90B05; 90B30; 90C30.

\section{Introduction}

Numerous types of production and inventory control models have been proposed in the literature. In general, different versions of these models are based on various combinations of typical simplifying assumptions. These assumptions include the following: constant demand rate, infinite production rate, constant unit purchase cost, constant unit holding cost, zero initial/end inventory, and minimum-cost objective. Although these assumptions generally facilitate the formulation and solution of such models, they also make these models less representative of real-life situations. By replacing these simplifying assumptions by realistic ones, a practical production and inventory control model is proposed in this paper. 
In many practical real-life situations, the demand for items is influenced by their in-stock availability. As frequently observed in the retail industry, the demand rate is an increasing function of the current inventory level. According to Sarkar [20], real-life examples of retail items with stock-dependent demand include sugar, spices, clothes, and gift cards. The stock-dependent demand phenomenon can be explained by several logical reasons. First, large displays and the physical presence of items is known to have a psychological influence that encourages customers' buying behavior. Second, customers tend to view higher item availability as an indication of continuous supply and on-time delivery. Although higher inventory levels lead to greater demand and increased revenue, they increase the holding cost. The proposed stock-dependent demand model aims to find optimum inventory levels while taking this trade-off into consideration. Therefore, the starting/ending inventory level of each cycle is considered as a decision variable to be optimally determined by the model. Allowing a non-zero starting/ending inventory is meant to increase the average inventory level, stimulating greater demand and higher sales revenue.

Inventory holding cost has several components, including opportunity loss, warehousing (space, labor, utilities, etc.), insurance, taxes, spoilage, and obsolescence. The main component is the investment opportunity loss or interest rate, because the assets invested in inventory cannot be invested to generate profits. In real-life inventory systems, storing items for extended time periods usually requires more advanced and expensive warehousing facilities. Therefore, variable holding cost models apply different unit holding costs to different ranges of storage-time durations. The proposed model assumes the holding cost to be a function of the unit purchase cost and the length of storage time. The model considers two types of holding cost time variability: retroactive and incremental. If the storage time extends over several holding cost ranges, incremental costing means each range has a different holding cost, while retroactive costing means the last range (highest) cost is used for all storage periods.

Suppliers frequently offer discounts for large orders in order to motivate customers to buy in larger quantities. Quantity-based discounts applicable to the unit purchase cost are classified into two main types: all-units, and marginal. In all-units discounts, the discounted cost is applied to all units in an order; while in marginal discounts, the discounted cost is applied only to the additional units above a certain amount. Since the all-units discount scheme is more common in real life, it is the type used in the model to be presented here. The model assumes a finite production rate and the gradual receipt of each order, as in the economic production quantity (EPQ) model. Finally, since the sales revenue is not constant, but is a function of the stock level, the objective of the model is to maximize the total profit instead of minimizing the total cost.

The remaining sections of this paper are organized as follows: relevant literature is reviewed in Section 2, the model is formulated in Section 3, solutions algorithms for both retroactive and incremental holding costs are presented in Sections 4 and 5, respectively. Finally, conclusions and future research suggestions are provided in Section 6. 


\section{Literature Review}

In this section, we review recent production planning models with the following relevant features: stock-dependent demand, variable holding costs, and quantity discounts. Several models have been proposed for items with stocklevel dependent demand rates. Teng and Chang [23] construct an economic production quantity (EPQ) model to maximize profit for deteriorating items, assuming the demand rate to be dependent on both the stock level and the unit sale price. For a deteriorating item with linearly stock-dependent demand in imprecise environments, Roy et al. [18] develop a model involving both fuzzy and random parameters, considering inflation and the time value of money. Hsieh and Dye [7] develop a deterministic economic order quantity (EOQ) model for deteriorating items with stock-dependent demand and finite shelf/display space, where shortages are allowed and unsatisfied demand is partially backlogged. Das et al. [4] present a production lot-size model in which the production rate has regular-time and overtime components, and the demand rate is stock-dependent. A single-objective optimization model is formulated to maximize average profit, and a genetic algorithm solution procedure is developed.

Duan et al. [5] present inventory models for perishable items with inventory level dependent demand, with and without backlogging. With backlogging, the backlogging rate is dependent on both backlogging time and amount. Without backlogging, stock remaining at the end of the cycle is sold for a lower salvage value. Assuming a stock-dependent demand rate, Sarkar [20] formulates a production model with a finite replenishment rate and imperfect production, i.e., the chance of producing defective items. In order to increase sales, buyers are offered a delay in payments under a progressive payment scheme. Recently, Yang et. al. [26] consider an EOQ model for a single manufacturer and a single retailer, where the demand rate at the retailer's end is dependent on the instantaneous stock level. The optimal credit period and quantity discount policy is determined, in order to maximize the total supply chain profit.

Quite a few production and inventory control models assume the holding cost is variable. Roy [17] constructs a deterministic inventory system where both the deterioration rate and the holding cost are linearly increasing functions of time, and the demand rate is a decreasing function of the selling price. Wahab and Jaber [25] analyze the effect of learning on the lot size for an EOQ model for items with imperfect quality, considering two different holding costs for the good and defective items. Mishra and Singh [12] formulate a deterministic deteriorating inventory model in which the demand rate and the holding cost are both linear functions of time. Assuming a constant deterioration rate and partial backordering with a variable backordering rate, an analytical model is developed to minimize the total inventory cost.

Gupta and Singh [6] develop a vendor-buyer production-inventory model with a variable deterioration rate, fuzzy demand and production rates, and variable holding cost. Two types of holding cost functions are considered: retroactive holding cost and incremental holding cost. Sazvar et al. [21] propose a continuous review system for modeling inventory control of perishable items 
under constant demand and stochastic supplier lead-time. Assuming uniform lead-time distribution, non-linear holding cost and complete backordering, an optimum solution procedure is developed. Pando et al. [14], [15] study an inventory model in which the demand rate is stock-dependent, the holding cost is nonlinear, and the objective is to maximize the average profit per unit time. Pando et al. [16] extend this stock-level dependent demand model, by considering the holding cost as a nonlinear function of both the inventory level and the storage time. Shah et al. [22] formulate an inventory model for a deteriorating item assuming an arbitrary deterioration rate and an arbitrary holding cost. An algorithm is proposed to maximize the total profit, given the demand rate is dependent on both the advertising level and the selling price.

Previous inventory models with quantity discounts address both all-units and incremental (marginal) quantity discounts. Benton and Park [3] present an overview of the quantity discounts research. Munson and Rosenblatt [13] present an exploratory study of 39 companies and their different discount strategies in practice. They find that $95 \%$ of these companies either offer or receive some type of all-units quantity discounts, while only $37 \%$ of them offer or receive incremental quantity discounts. $\mathrm{Hu}$ and Munson [8] present a heuristic for incremental quantity discounts with constant demand over a finite horizon. $\mathrm{Hu}$ et al. [9] suggest a modification of the classical Silver-Meal heuristic under the incremental quantity discount case to improve the results of $\mathrm{Hu}$ and Munson [8]. Mendoza and Ventura [11] develop an EOQ-type model with two modes of transportation, namely full-truckload and less-than-truckload carriers. Both all-units and incremental quantity discount structures are introduced into the analysis.

San-José and García-Laguna [19] consider an inventory model with full backordering of shortages and all-units quantity discounts. The backordering cost is assumed to consist of a fixed-cost component and a time-proportional component, and an algorithm based on the composite lot size model is developed to determine the optimal solution. Lin [10] proposes a buyer-supplier inventory model for items with imperfect quality, 100\% inspection, and quantity discounts. Assuming the buyer is more powerful, an optimum algorithm is developed to maximize the buyer's expected profit. Considering a supply chain context, Zhang, et al. [27] address buyer-vendor coordination for a product with a fixed lifetime. Assuming a finite production rate and deterministic demand, an integrated production-inventory model with quantity discounts is developed for both decentralized and centralized systems.

Alfares [1] develops EOQ-type models and algorithms for an inventory system with stock-dependent demand and variable holding costs. Urban [24] extends this model by allowing non-zero ending inventory and considering a profit-maximization objective. Alfares [2] extends that model to an economic manufacturing quantity (EMQ) framework, assuming a finite production rate. The objective is to minimize the total cost, assuming the starting/ending inventory level for each cycle to be zero. In this paper, the Alfares [2] model is extended in several ways, by considering quantity discounts, maximum-profit objective, and non-zero starting/ending inventory. Table 1 summarizes the features of the papers reviewed in this section. 
Table 1. Summary of features of the relevant papers reviewed in this paper*

\begin{tabular}{|c|c|c|c|c|c|c|c|c|c|}
\hline Paper & $\begin{array}{l}\text { Var. } \\
\text { dem }\end{array}$ & EOQ & EPQ & $\begin{array}{l}\text { Var. } \\
\text { hold }\end{array}$ & Disc & $\begin{array}{l}\text { Min } \\
\text { cost }\end{array}$ & $\begin{array}{l}\text { Max } \\
\text { profit }\end{array}$ & Deter & Short \\
\hline This paper & $\checkmark$ & & $\checkmark$ & $\checkmark$ & $\checkmark$ & & $\checkmark$ & & \\
\hline Alfares [1] & $\checkmark$ & $\checkmark$ & & $\checkmark$ & & $\checkmark$ & & & \\
\hline Alfares [2] & $\checkmark$ & & $\checkmark$ & $\checkmark$ & & $\checkmark$ & & & \\
\hline Das et al. [4] & $\checkmark$ & & $\checkmark$ & & & & $\checkmark$ & & \\
\hline Duan et al. [5] & $\checkmark$ & $\checkmark$ & & & & & $\checkmark$ & $\checkmark$ & $\checkmark$ \\
\hline Gupta and Singh $[6]$ & $\checkmark$ & & $\checkmark$ & $\checkmark$ & & $\checkmark$ & & $\checkmark$ & \\
\hline Hsieh and Dye [7] & $\checkmark$ & $\checkmark$ & & & & & $\checkmark$ & $\checkmark$ & $\checkmark$ \\
\hline $\mathrm{Hu}$ and Munson [8] & $\checkmark$ & $\checkmark$ & & & $\checkmark$ & $\checkmark$ & & & \\
\hline Hu et al. [9] & $\checkmark$ & $\checkmark$ & & & $\checkmark$ & $\checkmark$ & & & \\
\hline $\operatorname{Lin}[10]$ & & $\checkmark$ & & & $\checkmark$ & & $\checkmark$ & & \\
\hline Mendoza and Ventura [11] & & $\checkmark$ & & & $\checkmark$ & $\checkmark$ & & & \\
\hline Mishra and Singh [12] & $\checkmark$ & $\checkmark$ & & $\checkmark$ & & $\checkmark$ & & $\checkmark$ & $\checkmark$ \\
\hline Pando et al. [14], [15], [16] & $\checkmark$ & $\checkmark$ & & $\checkmark$ & & & $\checkmark$ & & \\
\hline Roy $[17]$ & $\checkmark$ & $\checkmark$ & & $\checkmark$ & & & $\checkmark$ & $\checkmark$ & $\checkmark$ \\
\hline Roy et al. [18] & $\checkmark$ & $\checkmark$ & & & & & $\checkmark$ & $\checkmark$ & \\
\hline $\begin{array}{l}\text { San-José and } \\
\text { García-Laguna [19] }\end{array}$ & & $\checkmark$ & & & $\checkmark$ & & $\checkmark$ & & $\checkmark$ \\
\hline Sarkar $[20]$ & $\checkmark$ & & $\checkmark$ & & & $\checkmark$ & & & \\
\hline Sazvar et al. [21] & & $\checkmark$ & & $\checkmark$ & & $\checkmark$ & & $\checkmark$ & $\checkmark$ \\
\hline Shah et al. [22] & $\checkmark$ & $\checkmark$ & & $\checkmark$ & & & $\checkmark$ & $\checkmark$ & \\
\hline Teng and Chang [23] & $\checkmark$ & & $\checkmark$ & & & & $\checkmark$ & $\checkmark$ & \\
\hline Urban [24] & $\checkmark$ & $\checkmark$ & & $\checkmark$ & & & $\checkmark$ & & \\
\hline Wahab and Jaber [25] & & $\checkmark$ & & $\checkmark$ & & $\checkmark$ & & & \\
\hline Yang et. al. [26] & $\checkmark$ & $\checkmark$ & & & $\checkmark$ & & $\checkmark$ & & \\
\hline Zhang, et al. [27] & & & $\checkmark$ & & $\checkmark$ & & $\checkmark$ & & \\
\hline
\end{tabular}

* Key

$\begin{array}{ll}\text { Var. dem } & =\text { variable demand } \\ \text { EOQ } & =\text { economic order quantity } \\ \text { EPQ } & =\text { economic production quantity } \\ \text { Var. hold } & =\text { variable holding cost } \\ \text { Disc } & =\text { quantity discounts } \\ \text { Min cost } & =\text { minimum-cost objective } \\ \text { Max profit } & =\text { maximum-profit objective } \\ \text { Deter } & =\text { deteriorating items } \\ \text { Short } & =\text { shortages are allowed }\end{array}$


Including all-units quantity discounts makes the model more practical, as these discounts are widely used in real life. Since the demand (revenue) is not constant, minimizing the cost is not an appropriate objective, as it does not necessarily lead to the maximum profit. Moreover, since the demand is dependent on the stock level, keeping a positive starting/ending inventory level will positively affect demand. Details of the new model are presented in the following section.

\section{Problem Description and Analysis}

The model considers a single item with an infinite planning horizon, where shortages are not allowed. The demand rate is an increasing power function of the inventory level, the holding cost rate is an increasing step function of storage duration and the unit purchase cost is a decreasing step function of the order lot size. The unit holding cost is a product of the unit purchase cost and the holding cost rate. The model assumes that the items are produced/received at a finite constant rate, and that these items contain no defects and do not deteriorate or lose value over time. Figure 1 presents the inventory level variation during the two phases of each production cycle. During the uptime phase $(0 \leq t \leq$ $\left.t_{1}\right)$, items are both produced and consumed. During the downtime phase $\left(t_{1}\right.$ $\leq t \leq T)$, items are only consumed as no production takes place.

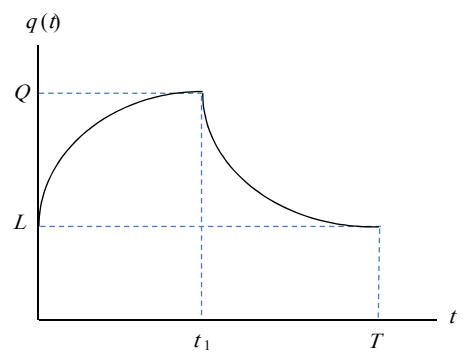

Figure 1. Inventory level variation during one production cycle.

The objective of the proposed production-inventory system is to find the maximum total profit per unit time. The profit depends on the following components: sales revenue, purchase cost, inventory holding cost, and ordering cost. Keeping a non-zero inventory at the start and end of each cycle increases the demand and hence the sales revenue, but it increases the holding cost. Therefore, the minimum (starting/ending) inventory level is a decision variable that is determined by the model.

\subsection{Notation}

\section{Model parameters}

$D=$ base demand rate, $D>0$;

$P=$ production rate during the first (uptime) phase of the cycle: $P>D$; 
$\alpha=D / P, 0<\alpha<1 ; \quad \beta=$ demand elasticity rate: $0<\beta<1$;

$\tau_{i}=$ end time of holding-cost interval $i\left(\tau_{i-1}<\tau_{i}\right), i=1, \ldots, m$, where $\tau_{0}=$ 0 , and $\tau_{m}=\infty$;

$h_{i}=$ holding cost per item unit per time unit during holding-cost interval $i$ $\left(h_{i-1}<h_{i}\right), i=1, \ldots, m$;

$\sigma_{j}=$ maximum lot size for purchase-cost range $j\left(\sigma_{j-1}<\sigma_{i}\right), j=1, \ldots, n$, where $\sigma_{0}=0$, and $\sigma_{n}=\infty$;

$c_{j}=$ unit purchase cost in purchase-cost range $j\left(c_{j-1}<c_{i}\right), j=1, \ldots, n$;

$K=$ ordering cost per order, $K \geq 0 ; \delta=$ sale price per unit of the item, $\delta>0$.

\section{Auxiliary variables}

$t=$ current time, i.e. time from the start of the cycle, $t \geq 0$;

$t_{1}=$ end time of the first (uptime) phase of the cycle, $0<t_{1}<T$;

$T=$ cycle time, i.e. time between producing two consecutive orders;

$S=$ production lot size: $S=P t_{1}$;

$X_{i}=\left\{\begin{array}{l}1, \text { if } \tau_{i-1} \leq T \leq \tau_{i}, \quad i=1, \ldots, m ; \\ 0, \text { otherwise, }\end{array}\right.$

$Y_{j}=\left\{\begin{array}{l}1, \text { if } \sigma_{j-1} \leq S \leq \sigma_{j}, \quad j=1, \ldots, n . \\ 0, \text { otherwise, }\end{array}\right.$

\section{Model functions}

$q(t)=$ inventory level at current time $t$;

$R[q(t)]=$ stock-dependent demand rate: $R[q(t)]=D[q(t)]^{\beta}$;

$h(t)=$ holding cost rate (per unit item per time period) during time $t$;

$h(t)=h_{i}$ if $\tau_{i-1} \leq t \leq \tau_{i}\left(h_{1}<h_{2}<\ldots<h_{m}\right)$;

$c(S)=$ purchase cost per unit of the item if the lot size is equal to $S$;

$c(S)=c_{j}$ if $\sigma_{j-1} \leq S \leq \sigma_{j}\left(c_{1}>c_{2}>\ldots>c_{n}\right)$.

\section{Decision variables}

$Q=$ maximum inventory level, corresponding to time $t=t_{1}$ :

$Q=\max \{q(t)\}=q\left(t_{1}\right)$;

$L=$ starting/ending inventory level, corresponding to times $t=0$ and $t=T$ :

$L=\min \{q(t)\}=q(0)=q(T)$.

\section{$3.2 \quad$ Uptime phase analysis}

During this phase of the production-inventory cycle, both production and consumption take place at the same time. While the inventory level increases at the constant production rate of $P$, it simultaneously decreases at the stockdependent demand rate of $D[q(t)]^{\beta}$. Accordingly, the inventory level is described by the following differential equation:

$$
\frac{d q(t)}{d t}=P-D q(t)^{\beta}, \quad 0 \leq t \leq t_{1}, \quad P>D .
$$

Rearranging and integrating the ordinary differential equation (3.1) results in the following equation:

$$
\int_{0}^{t} \frac{1}{1-\alpha q(t)^{\beta}} d q(t)=P \int_{0}^{t} d t
$$


The left-hand side of (3.2) is integrated to yield the hyper-geometric function ${ }_{2} F_{1}$ :

$$
\int \frac{1}{1-\alpha q^{\beta}} d q=q \times\left[{ }_{2} F_{1}\left(1, \frac{1}{\beta} ; 1+\frac{1}{\beta} ; \alpha q^{\beta}\right)\right]
$$

where

$$
{ }_{2} F_{1}\left(1, \frac{1}{\beta} ; 1+\frac{1}{\beta} ; \alpha q^{\beta}\right)=\sum_{n=0}^{\infty} \frac{(n !)\left(\frac{1}{\beta}\right)\left(\frac{1}{\beta}+1\right) \ldots\left(\frac{1}{\beta}+n-1\right)}{\left(\frac{1}{\beta}+1\right) \ldots\left(\frac{1}{\beta}+n-1\right)\left(\frac{1}{\beta}+n\right)} \frac{\left(\alpha q^{\beta}\right)^{n}}{n !}=\sum_{n=0}^{\infty} \frac{\alpha^{n} q^{n \beta}}{(n \beta+1)} .
$$

Therefore, (3.2) can be written as follows:

$$
\left.\sum_{n=0}^{\infty} \frac{\alpha^{n} q(t)^{n \beta+1}}{(n \beta+1)}\right|_{a} ^{b}=\left.P t\right|_{a} ^{b}, \quad 0 \leq t \leq t_{1} .
$$

Since $q(0)=L$, integrating $(3.3)$ over the range $[0, t]$ gives:

$$
\sum_{n=0}^{\infty} \frac{\alpha^{n}\left[q(t)^{n \beta+1}-L^{n \beta+1}\right]}{P(n \beta+1)}=t .
$$

Substituting $q(0)=L$ and $q\left(t_{1}\right)=Q$, integrating (3.3) over the range $\left[0, t_{1}\right]$ gives:

$$
t_{1}=\sum_{n=0}^{\infty} \frac{\alpha^{n}\left(Q^{n \beta+1}-L^{n \beta+1}\right)}{P(n \beta+1)} .
$$

In order to obtain an expression for $q(t)$ during the uptime phase, (3.1) is rearranged differently from (3.2) and integrated as follows:

$$
\int_{a}^{b} q(t) d t=\frac{1}{P} \int_{a}^{b} \frac{q(t)}{1-\alpha q(t)^{\beta}} d q .
$$

The right-hand side of (3.6) again integrates to the hyper-geometric function ${ }_{2} F_{1}$ :

$$
\int \frac{q}{1-\alpha q^{\beta}} d q=\frac{q^{2}}{2} \times_{2} F_{1}\left(1, \frac{2}{\beta} ; 1+\frac{2}{\beta} ; \alpha q^{\beta}\right) .
$$

After simplification, the uptime phase equation (3.5) can be written as:

$$
\int_{a}^{b} q(t) d t=\sum_{n=0}^{\infty} \frac{\alpha^{n}\left[q(b)^{n \beta+2}-q(a)^{n \beta+2}\right]}{P(n \beta+2)} .
$$

\subsection{Downtime phase analysis}

During this phase of the cycle, the production is down, and the inventory is consumed according to the stock level-dependent demand rate. The applicable differential equation to describe the inventory level is:

$$
\frac{d q(t)}{d t}=-D q(t)^{\beta}, \quad t_{1} \leq t \leq T .
$$


The solution of the above ordinary equation is shown by Alfares [1] for the case of zero starting/ending inventory $(L=0)$. Revising the solution to allow a non-zero starting/ending inventory $(L \geq 0)$ leads to the following results:

$$
\begin{aligned}
& q(t)=\left[Q^{1-\beta}-D(1-\beta)\left(t-t_{1}\right)\right]^{\frac{1}{1-\beta}}, \quad t_{1} \leq t \leq T \\
& \int_{a}^{b} q(t) d t=\frac{q(a)^{2-\beta}-q(b)^{2-\beta}}{D(2-\beta)} \\
& T=t_{1}+\frac{Q^{1-\beta}-L^{1-\beta}}{D(1-\beta)}
\end{aligned}
$$

From the optimum values of $Q$ and $L$, the following quantities can be calculated:

$$
\int_{0}^{T} q(t) d t=\frac{Q^{2-\beta}-L^{2-\beta}}{D(2-\beta)}+\sum_{n=0}^{\infty} \frac{\alpha^{n}\left(Q^{n \beta+2}-L^{n \beta+2}\right)}{P(n \beta+2)}, \quad S=P t_{1} .
$$

Convergence of the calculations, i.e. obtaining finite values for the summation terms, is guaranteed by satisfying the condition $\left(\alpha Q^{\beta}<1\right)$, which can be expressed as follows:

$$
Q<\alpha^{-1 / \beta}
$$

Usually, the upper bound on $Q$ specified in (3.12) is so high that it cannot realistically be reached. The bound in (3.12) should be compared with $Q_{\max }$, the maximum possible $Q$ obtained by substituting the minimum cost values on the model. If the bound in (3.12) is much larger than $Q_{\max }$, then constraint (3.12) is considered redundant and can be safely removed from the model.

The net profit for the production-inventory system is equal to the total revenue resulting from the sale of manufactured products, minus the costs of purchasing, ordering, and holding. The sales revenue per cycle is equal to the sale price per unit $(\delta)$ times the number of units sold $\left(S=P t_{1}\right)$. The purchasing cost in each cycle is equal to the purchase cost per unit $\left(c_{j}\right)$ times the number of units sold $\left(P t_{1}\right)$. The ordering cost for each cycle is simply equal to $K$. The total holding cost for a given cycle is obtained by integrating the product of the applicable holding cost rate $\left(h_{i} c_{j}\right)$ and the corresponding inventory level $q(t)$ over the interval $[0, T]$. Combining the terms and dividing by the cycle time $T$, the net profit per unit time $\pi_{i, j}(Q, L)$ is given by:

$$
\pi_{i, j}(Q, L)=\frac{1}{T}\left[\delta P t_{1}-c_{j} P t_{1}-K-h_{i} c_{j} \int_{0}^{T} q(t) d t\right] .
$$

Obviously, since (3.5) and (3.11) respectively specify $t_{1}$ and $\int_{0}^{T} q(t) d t$ to be functions of $Q$ and $L$, then the objective (3.13) is only a function of the decision variables $Q$ and $L$. The feasible region for this function is the triangular region $\Omega=\left\{(\mathrm{Q}, \mathrm{L}) \in \mathbb{R}^{2} / 0<Q<\alpha^{-1 / \beta}, 0 \leq L<Q\right\}$. Discretely variable values of $h_{i}$ and $c_{j}$ are applicable for different ranges of storage times $t$ and order sizes $S$. Therefore, the profit function defined in (3.13) is discontinuous at storage time breakpoints $\tau_{i}$, and order size breakpoints $\sigma_{j}$. Additional properties of the objective function (3.13), namely its sensitivity to changes in input parameters, are discussed in section 4.3 and 5.4 


\section{Retroactive holding cost case}

\subsection{Retroactive holding cost model}

Under a retroactive holding cost structure, one holding cost $\left(h_{i}\right)$ is applicable to all storage periods. If the cycle ends in interval $i,\left(\tau_{i-1} \leq T<\tau_{i}\right)$, then the rate $\left(h_{i}\right)$ is applied to all holding cost intervals: $1,2, \ldots, i$. Moreover, given the lot size is in range $j\left(\sigma_{j-1} \leq S<\sigma_{j}\right)$, then one unit purchase cost $\left(c_{j}\right)$ is applied to all units in an order (all-units discounts). In this section, a nonlinear programming (NLP) model is formulated to determine the applicable combination of $h_{i}$ and $c_{j}$, and the corresponding optimum values of the decision variables: $t_{1}, T, Q$, and $L$. The objective function of the NLP model is obtained by replacing the constants $\left(h_{i}\right.$ and $\left.c_{j}\right)$ in (3.13) by $0-1$ linear combinations of all possible values of $h_{i}$ and $c_{j}$, allowing the model to select their optimum values. The objective, which is to maximize the total profit per unit time, is expressed as follows: Maximize

$$
\pi_{i, j}=\frac{1}{T}\left[P t_{1} \delta-P t_{1}\left(\sum_{j=1}^{n} c_{j} Y_{j}\right)-K-\left(\sum_{j=1}^{n} c_{j} Y_{j}\right)\left(\sum_{i=1}^{m} h_{i} X_{i}\right) \int_{0}^{T} q(t) d t\right] .
$$

In order to select the correct values $h_{i}$ and $c_{j}$ for the given intervals of $T$ and $S$, respectively, the objective function (4.1) is optimized subject to the following constraints:

$$
\begin{aligned}
& \sum_{i=1}^{m} X_{i}=1, \\
& \sum_{j=1}^{n} Y_{j}=1, \\
& \sum_{i=1}^{m} \tau_{i} X_{i} \geq T, \\
& \sum_{j=1}^{n} \sigma_{j-1} Y_{j} \leq S .
\end{aligned}
$$

Constraint (4.2) ensures that only one value is selected for the unit holding cost $h_{i}$, while constraint (4.3) ensures that only one value is selected for the unit purchase cost $c_{j}$. Constraint (4.4) ensures that the right binary $X_{j}$ variable (corresponding to the right interval of $T$ ) is selected for the unit holding cost $h_{i}$, while constraint (4.5) ensures that the right binary $Y_{j}$ variable (corresponding to the right range of $S$ ) is selected for the unit purchase cost $c_{j}$. Constraints (4.2) and (4.4) are applicable to the unit holding cost $h_{i}$, which is an increasing step function of $T$. On the other hand, constraints (4.3) and (4.5) are applicable to the unit purchase cost $c_{j}$, which is a decreasing step function of $S$. As far as the author knows, these logical constraints for both increasing and decreasing step functions are new formulations, first introduced in this paper.

In addition to logical constraints (4.2) through (4.5), the objective (4.1) is also subjected to inventory cycle constraints (3.5), (3.10), (3.11), and (3.12). 
The NLP model of the retroactive holding cost case can be efficiently solved by conventional NLP techniques and software. A solved example illustrating the model's formulation and solution is presented in the following section.

\subsection{Retroactive holding cost example}

Given the following parameter values: $D=400$ units per year, $P=1000$ units per year, $K=\$ 300$ per order, $h_{1}=\$ 12 \%$ per year, $h_{2}=\$ 16 \%$ per year, $h_{3}=$ $20 \%$ per year, $c_{1}=50, c_{2}=45, c_{3}=40, \tau_{1}=0.3$ years, $\tau_{2}=0.6$ years, $\beta=$ $0.1, \sigma_{1}=300, \sigma_{2}=500, \delta=\$ 70 /$ unit.

The optimum solution is obtained in the following steps:

$$
\alpha=400 / 1000=0.4, \quad \alpha^{-1 / \beta}=0.4^{-10}=9536 .
$$

Since the upper limit $(Q=9536)$ is much larger than the maximum possible value for $Q\left(Q_{\max }=704\right.$, as shown in section 5.3), we may remove (3.12) from the NLP model.

Assuming a retroactive holding cost, the NLP model is formulated by substituting the given parameter values in the objective (4.1) and constraints (4.2) through (4.5), (3.5), (3.10), and (3.11), as shown below: Maximize

$$
\pi_{i, j}(Q, L)=\frac{1}{T}\left[\begin{array}{l}
1000(70) t_{1}-1000 t_{1}\left(50 Y_{1}+45 Y_{2}+40 Y_{3}\right)-300 \\
-\left(50 Y_{1}+45 Y_{2}+40 Y_{3}\right)\left(0.12 X_{1}+0.16 X_{2}+0.20 X_{3}\right) \int_{0}^{T} q(t) d t
\end{array}\right],
$$

subject to:

$$
\begin{aligned}
& X_{1}+X_{2}+X_{3}=1, \quad 0.3 X_{1}+0.6 X_{2}+N X_{3} \geq T \\
& Y_{1}+Y_{2}+Y_{3}=1, \quad 0 Y_{1}+300 Y_{2}+500 Y_{3} \leq 1000 t_{1}, \\
& t_{1}=\sum_{n=0}^{\infty} \frac{0.4^{n}\left(Q^{0.1 n+1}-L^{0.1 n+1}\right)}{1000(0.1 n+1)}, \\
& T=t_{1}+\frac{Q^{0.9}-L^{0.9}}{400(0.9)}, \\
& \int_{0}^{T} q(t) d t=\frac{Q^{1.9}-L^{1.9}}{400(1.9)}+\sum_{n=0}^{\infty} \frac{0.4^{n}\left(Q^{0.1 n+2}-L^{0.1 n+2}\right)}{1000(0.1 n+2)} .
\end{aligned}
$$

Before solving the above NLP model, each $(\infty)$ value in (4.6), (4.9), and (4.10) has been replaced by a very large number, $N$. Based on numerical experiments, substituting a value of $N=2000$ for $\infty$ is sufficient to sustain numerical stability and achieve fully accurate results. The above NLP model is near-optimally solved using the evolutionary search method of Microsoft Excel's Solver. To maximize the probability of an optimal solution, Excel Solver was run 10 times for each NLP model, and the best result was chosen. Differences between the objective functions of the different runs were very slight, and usually only seen in the decimal portion of the result. The optimum solution of the above NLP model is given by:

$$
L=134, Q=440, t_{1}=1.0300, T=1.4669, S=1030, \pi(Q, L)=18533.75 .
$$


In order to compare with the Alfares [2] model, the above example was solved assuming $L=0$ and the objective is to minimize the total cost per unit time. Assuming a retroactive holding cost, the Alfares [2] model provides the following solution $\left(L=0, Q=135, t_{1}=0.338, T=0.567, S=337\right)$. Since the Alfares model does not consider quantity discounts, a uniform no-discount purchase price $\left(c_{1}=50\right)$ is assumed, leading to a total profit per unit time equal to $\pi_{i, j}(Q, L)=10805.24$. If we consider quantity discounts corresponding to the production lot size $(S=337)$ and use a unit purchase cost of $\left(c_{2}=45\right)$, then the total profit per unit time increases to $\pi_{i, j}(Q, L)=13830.99$. Comparing 13830.99 to 18533.75 , the new model increases the profit per period by $34 \%$. It is clear that the model presented in this paper produces a much higher profit, even if quantity discounts are added to the Alfares [2] model.

\subsection{Sensitivity analysis}

In order to analyze the sensitivity of the model to changes in input parameters ( $\left.D, P, K, \delta, h_{i}, c_{j}\right)$, a sensitivity analysis was conducted on the above example. One at a time, each input parameter was individually changed by steps of $-20 \%,-10 \%,+10 \%$, and $+20 \%$, and changes in the final solution were observed. A total of 24 problems were solved to perform the sensitivity analysis, and the results are summarized in Table 2. The model's sensitivity to changes in the input parameters is mainly indicated by the range of the objective values $\pi_{i, j}(Q, L)$ in Table 2 . As expected, the total profit per unit time $\pi_{i, j}(Q, L)$ is positively correlated with the demand rate $D$ and the selling price $\delta$, and negatively correlated with all the cost parameters $\left(K, h_{i}, c_{j}\right)$. Interestingly, however, the profit is also negatively correlated with the production rate $P$. The value of $\pi_{i, j}(Q, L)$ is most sensitive to changes in the selling price and least sensitive to changes in the ordering cost $K$.

\section{Incremental Holding Cost}

\subsection{Incremental holding cost model}

If the inventory holding cost increases incrementally, then different holding cost rates $h_{i}$ are used for each storage interval $i\left(\tau_{i-1} \leq t<\tau_{i}\right)$. Therefore, assuming an incremental holding cost, the maximum-profit objective function (4.1) is modified as follows: Maximize

$$
\pi_{i, j}(Q, L)=\frac{1}{T}\left[P t_{1} \delta-P t_{1} \sum_{j=1}^{n} c_{j} Y_{j}-K-\sum_{j=1}^{n} c_{j} Y_{j} \sum_{i=1}^{e} h_{i} \int_{\tau_{i-1}}^{\tau_{i}} q(t) d t\right] .
$$

The symbol $e$ is introduced above to represent the ending holding-cost interval in which $T$ is located $\left(\tau_{e-1} \leq T<\tau_{e}\right)$. For this last holding-cost interval, $e$, in which the cycle ends, we set $\tau_{i}=T$ in (5.1). In order to apply (5.1) correctly, the forms of integrals $\int_{\tau_{i-1}}^{\tau_{i}} q(t) d t$ are determined from different expressions in the two phases of the production-inventory cycle. The forms of $\int_{\tau_{i-1}}^{\tau_{i}} q(t) d t$ are developed from (3.7) for the uptime phase (before $t_{1}$ ) and from (3.9) for the 
Table 2. Sensitivity analysis for the retroactive holding cost example.

\begin{tabular}{|c|c|c|c|c|c|c|c|c|}
\hline Parameter & Original value & New values & $L$ & $Q$ & $t_{1}$ & $T$ & $S$ & $\pi_{i, j}(Q, L)$ \\
\hline \multirow[b]{4}{*}{$D$} & \multirow[b]{4}{*}{400} & 320 & 101 & 393 & 0.653 & 1.182 & 653 & 14326.73 \\
\hline & & 360 & 118 & 414 & 0.794 & 1.267 & 794 & 16413.37 \\
\hline & & 440 & 166 & 469 & 1.391 & 1.780 & 1391 & 20686.54 \\
\hline & & 480 & 326 & 396 & 0.518 & 0.599 & 518 & 23072.42 \\
\hline \multirow[b]{4}{*}{$P$} & \multirow[b]{4}{*}{1000} & 800 & 161 & 387 & 2.311 & 2.635 & 1849 & 18672.15 \\
\hline & & 900 & 143 & 422 & 1.414 & 1.813 & 1273 & 18590.35 \\
\hline & & 1100 & 128 & 459 & 0.835 & 1.307 & 919 & 18490.98 \\
\hline & & 1200 & 129 & 479 & 0.708 & 1.206 & 850 & 18456.30 \\
\hline \multirow[b]{4}{*}{$K$} & \multirow[b]{4}{*}{300} & 240 & 145 & 436 & 0.983 & 1.398 & 983 & 18576.28 \\
\hline & & 270 & 140 & 430 & 0.975 & 1.389 & 975 & 18554.72 \\
\hline & & 330 & 132 & 448 & 1.066 & 1.517 & 1066 & 18512.75 \\
\hline & & 360 & 130 & 456 & 1.106 & 1.572 & 1106 & 18492.29 \\
\hline \multirow[b]{4}{*}{$\delta$} & \multirow[b]{4}{*}{70} & 56 & 48 & 268 & 0.648 & 0.983 & 648 & 8956.73 \\
\hline & & 63 & 83 & 367 & 0.902 & 1.319 & 902 & 13676.40 \\
\hline & & 77 & 194 & 520 & 1.160 & 1.615 & 1160 & 23508.27 \\
\hline & & 84 & 255 & 600 & 1.289 & 1.761 & 1289 & 28581.44 \\
\hline \multirow[b]{4}{*}{$h_{1}, h_{2}, h_{3}$} & \multirow[b]{4}{*}{$0.12,0.16,0.20$} & $0.10,0.13,0.16$ & 338 & 720 & 1.518 & 2.029 & 1518 & 19094.01 \\
\hline & & $0.11,0.145,0.18$ & 160 & 478 & 1.099 & 1.548 & 1099 & 18780.75 \\
\hline & & $0.13,0.175,0.22$ & 121 & 403 & 0.929 & 1.335 & 929 & 18310.49 \\
\hline & & $0.14,0.19,0.24$ & 101 & 376 & 0.886 & 1.286 & 886 & 18106.26 \\
\hline \multirow[b]{4}{*}{$c_{1}, c_{2}, c_{3}$} & \multirow[b]{4}{*}{$50,45,40$} & $40,36,32$ & 275 & 645 & 1.411 & 1.914 & 1411 & 24889.34 \\
\hline & & $45,40.5,36$ & 200 & 536 & $\begin{array}{l}1.411 \\
1.205\end{array}$ & $\begin{array}{l}1.914 \\
1.672\end{array}$ & $\begin{array}{l}1411 \\
1205\end{array}$ & 21641.98 \\
\hline & & $55,49.5,44$ & 92 & 362 & 0.860 & 1.255 & 860 & 15546.03 \\
\hline & & $60,54,48$ & 62 & 311 & 0.759 & 1.132 & 759 & 12669.03 \\
\hline
\end{tabular}

downtime phase (after $t_{1}$ ). If a given interval $\left[\tau_{i-1}, \tau_{i}\right]$ overlaps both phases, then it is divided into two parts, $\left[\tau_{i-1}, t_{1}\right]$ and $\left[t_{1}, \tau_{i}\right]$. In that case, for $\tau_{i-1} \leq t_{1} \leq \tau_{i}$ the integral is obtained by combining (3.7), assuming $q(b)=$ $q\left(t_{1}\right)=Q$, and (3.9), assuming $q(a)=q\left(t_{1}\right)=Q$, as follows:

$$
\int_{\tau_{i-1}}^{\tau_{i}} q(t) d t=\sum_{n=0}^{\infty} \frac{\alpha^{n}\left[Q^{n \beta+2}-q\left(\tau_{i-1}\right)^{n \beta+2}\right]}{P(n \beta+2)}+\frac{Q^{2-\beta}-q\left(\tau_{i}\right)^{2-\beta}}{D(2-\beta)} .
$$

It is clear from (3.7), (3.9), and (5.2) that inventory levels at the end of each holding cost interval $q\left(\tau_{i}\right)$ are needed to calculate all the integrals $\int_{\tau_{i-1}}^{\tau_{i}} q(t) d t$. Therefore, for each boundary point $\tau_{i}\left(0<\tau_{i}<T\right)$, we need to add a constraint to calculate the corresponding inventory level $q\left(\tau_{i}\right)$, using $(3.4)$ for the uptime phase and (3.8) for the downtime phase. For the last holding-cost interval $e$, $\tau_{i}=T$, and thus the boundary value $q\left(\tau_{i}\right)=q(T)=L$ is substituted in (3.9).

As discussed above, the locations of $t_{1}$ and $T$ with respect to holding cost intervals $\left(\tau_{i-1}, \tau_{i}\right)$ need to be pre-determined in order to formulate (5.1). To determine the range of holding cost intervals in which $t_{1}$ and $T$ are located, we first solve the NLP using the minimum values of both $h_{i}$ and $c_{j}$ in equation (5.1): Maximize

$$
\pi_{i, j}(Q, L)=\frac{1}{T}\left[P t_{1} \delta-P t_{1} c_{\min }-K-c_{\min } h_{\min } \int_{0}^{T} q(t) d t\right] .
$$


The objective function (5.3) is maximized subject to constraints (3.5), (3.10), (3.11), and (3.12). From the solution of this initial NLP model, we find the upper limits on the values of $t_{1}$ and $T$, and the corresponding pair of holding cost intervals $\left(\tau_{i-1}, \tau_{i}\right)$ in which they are located.

For each possible pair of holding cost intervals for $t_{1}$ and $T$, we construct the corresponding incremental-cost NLP model by maximizing the objective function (5.1) subject to constraints (3.5), (3.10), (3.12), (4.3), and (4.5), as well as applicable constraints on $\int_{\tau_{i-1}}^{\tau_{i}} q(t) d t$ from $(3.7),(3.9)$, and (5.2). In addition, for each $\tau_{i}\left(0<\tau_{i}<T\right)$ we include a constraint from (3.4) in the uptime phase and from (3.8) in the downtime phase.

\subsection{Incremental holding cost solution algorithm}

The above-described NLP model for the incremental holding cost case is very difficult to solve optimally. This difficulty is caused by the non-smooth, highly nonlinear objective function and constraints, the discontinuous (step) functions of both the holding cost and the purchase cost, and the existence of the 0-1 integer variables $X_{i}$ and $Y_{j}$. If integrality constraints are also added for the decision variables $Q$ and $L$, then the NLP model becomes practically unsolvable by conventional optimization techniques and software. Therefore, an intelligent search procedure is developed to efficiently obtain optimal solutions for this formidable NLP model. The search procedure is intelligent because it is designed for this particular problem, and it is built on logic that guides the moves towards the optimum solution. The steps of this solution procedure are described below.

1. Solve the NLP model using the minimum values of $h_{i}$ and $c_{j}$ in (5.3) subject to constraints $(3.5),(3.10),(3.11)$, and (3.12) to determine upper limits on $t_{1}$ and $T$. The pair of holding cost intervals in which $t_{1}$ and $T$ are respectively located is the starting holding-cost-interval pair for $t_{1}$ and $T$.

2. For the current holding-cost-interval pair for $t_{1}$ and $T$, construct the corresponding NLP model. Maximize (5.1) subject to (3.5), (3.10), (3.12), (4.3), and (4.5), in addition to applicable constraints on $\int_{\tau_{i-1}}^{\tau_{i}} q(t) d t$ from $(3.7)$, (3.9), and (5.2), and also applicable constraints on $\tau_{i}$ from (3.4) and (3.8). Initialize the value of $L$.

3. For the current value of $L$, and for each $\left(\tau_{i}\right)$ in the uptime phase $\left(0<\tau_{i}<\right.$ $\left.t_{1}\right)$, calculate the value of $q\left(\tau_{i}\right)$ that satisfies the corresponding constraint of the form (3.4), i.e., that makes the right-hand side equal to $\tau_{i}$. Fixing $L$, calculate the value of $q\left(\tau_{1}\right)$. Fixing $L$ and $q\left(\tau_{1}\right)$, calculate the value of $q\left(\tau_{2}\right)$, and so on.

4. Fixing the values of $L$ and all $q\left(\tau_{i}\right), 0<\tau_{i}<t_{1}$, search for the value of $Q$ that maximizes the objective (5.1), i.e., profit $\pi_{i, j}(Q, L)$. Starting with $Q$ $=\max \left\{q\left(\tau_{i}\right)\right\}$, increase the value of $Q$ until the profit $\pi_{i, j}(Q, L)$ begins to decrease or either $t_{1}$ or $T$ fall outside their current holding-cost intervals. 
5. If the current objective function $\pi_{i, j}(Q, L)$ is greater than the previous value, then increase $L$ and repeat steps $3-4$. If $\pi_{i, j}(Q, L)$ has decreased, then identify the previous solution as the best solution for the current holding-cost-interval pair of $t_{1}$ and $T$, and go to step 6 .

6. If the current pair of $t_{1}$ and $T$ intervals has a higher profit value $\pi_{i, j}(Q, L)$ than the previous pair, generate a new pair by moving $t_{1}$ or $T$ to an earlier interval, and then go to step 2. Otherwise, the best solution of the previous pair, found in step 5 , is identified as the final optimum solution.

\subsection{Incremental holding cost example}

The same parameter values given in the example of section 4.2 are used, but now an incremental holding cost is assumed.

Step 1:

Substituting minimum holding and purchase costs in (5.3), the objective function of the initial NLP model is shown below.

Maximize

$$
\pi_{i, j}(Q, L)=\frac{1}{T}\left[1000(70) t_{1}-1000(40) t_{1}-300-40(0.12) \int_{0}^{T} q(t) d t\right] .
$$

Maximizing this objective function subject to constraints (4.8)-(4.10), the optimum solution is given by:

$$
\begin{aligned}
& L=281, Q=702, t_{1}=1.635667, T=2.204027, S=1635.667, \\
& \pi_{i, j}(Q, L)=19740.86 .
\end{aligned}
$$

Since this solution is based on the minimum cost values, it provides upper limits on the values given above. According to the above solution, the third holding-cost interval $(t>0.6)$ is the latest possible interval for both $t_{1}$ and $T$. Therefore, this is the first $\left(t_{1}, T\right)$ holding-cost interval pair to be considered. (a) Both $t_{1}$ and $T$ are in the third interval.

Step 2a:

Assuming $t_{1}$ and $T$ are both in the third holding-cost interval $\left(t_{1}>0.6, T>\right.$ $0.6)$, then the lot size $S$ has to be the third purchase-cost range $(S>500)$ since $S=P t_{1}>1000(0.6)$. Accordingly, the objective function (5.1) is formulated as follows: Maximize

$$
\pi_{i, j}(Q, L)=\frac{1}{T}\left[\begin{array}{l}
1000(70) t_{1}-1000(40) t_{1}-300 \\
-40\left(0.12 \int_{0}^{0.3} q(t) d t+0.16 \int_{0.3}^{0.6} q(t) d t+0.2 \int_{0.6}^{T} q(t) d t\right)
\end{array}\right] .
$$

This objective function is maximized subject to constraints (4.7) through (4.9), in addition to the following constraints. First, using (3.7), the uptime phase integrals for the first and the second holding-cost intervals are expressed as follows:

$$
\int_{0}^{0.3} q(t) d t=\sum_{n=0}^{\infty} \frac{0.4^{n}\left[q(0.3)^{n \beta+2}-L^{n \beta+2}\right]}{1000(0.1 n+2)},
$$




$$
\int_{0.3}^{0.6} q(t) d t=\sum_{n=0}^{\infty} \frac{0.4^{n}\left[q(0.6)^{n \beta+2}-q(0.3)^{n \beta+2}\right]}{1000(0.1 n+2)} .
$$

The integral for the third holding-cost interval, overlapping the uptime and downtime phases, is formulated from (5.2) as follows:

$$
\int_{0.6}^{T} q(t) d t=\sum_{n=0}^{\infty} \frac{0.4^{n}\left[Q^{0.1 n+2}-q(0.6)^{0.1 n+2}\right]}{1000(0.1 n+2)}+\frac{Q^{1.9}-L^{1.9}}{400(1.9)}
$$

Finally, (3.4) is used to develop boundary-value constraints for $q(t)$ at interval border points $\tau_{1}=0.3$ and $\tau_{2}=0.6$, both in the uptime phase:

$$
\begin{aligned}
& \sum_{n=0}^{\infty} \frac{0.4^{n}\left[q(0.3)^{0.1 n+1}-L^{0.1 n+1}\right]}{1000(0.1 n+1)}=0.3 \\
& \sum_{n=0}^{\infty} \frac{0.4^{n}\left[q(0.6)^{0.1 n+1}-L^{0.1 n+1}\right]}{1000(0.1 n+1)}=0.6 .
\end{aligned}
$$

Steps 3a-4a:

Optimum solutions for representative values of $L$ are shown in Table 3.

Table 3. Optimum solutions for representative values of $L$, with both $t_{1}$ and $T$ in the third interval.

\begin{tabular}{llllllll}
\hline$L$ & $q(0.3)$ & $q(0.6)$ & $Q$ & $t_{1}$ & $T$ & $S$ & $\pi_{i, j}(Q, L)$ \\
\hline 50 & 159.448 & 254.974 & 255 & 0.600088 & 0.913155 & 600 & 18369.63 \\
\hline 100 & 202.009 & 293.801 & 294 & 0.600678 & 0.888017 & 600 & 18663.48 \\
\hline 150 & 246.442 & 334.875 & 335 & 0.600441 & 0.868269 & 600 & 18829.79 \\
\hline 200 & 291.956 & 377.372 & 378 & 0.602274 & 0.855233 & 602 & 18918.63 \\
\hline 250 & 338.183 & 420.876 & 421 & 0.600462 & 0.839736 & 600 & 18955.72 \\
\hline 300 & 384.921 & 465.135 & 466 & 0.60332 & 0.832464 & 603 & 18950.67 \\
\hline 350 & 432.043 & 509.984 & 510 & 0.600065 & 0.818333 & 600 & 18920.34 \\
\hline 400 & 479.464 & 555.308 & 556 & 0.602798 & 0.813338 & 602 & 18859.94 \\
\hline
\end{tabular}

Step 5a:

Conducting a focused search in the region $L=250-300$ leads to the following best solution for the case when both $t_{1}$ and $T$ are in the third interval:

$$
\begin{aligned}
& L=275, q(0.3)=361.498, q(0.6)=442.923, Q=443, t_{1}=0.600292, \\
& T=0.833737, S=600, \pi_{i, j}(Q, L)=18958.70 .
\end{aligned}
$$

\section{Step 6a}

Since there is no better previous solution, we continue to the next pair of holding-cost intervals for $t_{1}$ and $T$ by moving $t_{1}$ from the third to the second interval. 
(b). $t_{1}$ is in the second interval and $T$ is in the third interval.

Step 2b:

Assuming $t_{1}$ in the second holding-cost interval $\left(0.3 \leq t_{1} \leq 0.6\right), T$ in the third interval $(T>0.6)$, and the lot size is in the third purchase-cost range, the NLP model is formulated as follows: the objective function (5.4) is maximized subject to constraints (4.8), (4.9), (5.5), and (5.6), in addition to the following constraints.

The integral for the second interval, overlapping the uptime and downtime phased, is formulated from (5.2) as follows:

$$
\int_{0.3}^{0.6} q(t) d t=\sum_{n=0}^{\infty} \frac{0.4^{n}\left[Q^{0.1 n+2}-q(0.3)^{0.1 n+2}\right]}{1000(0.1 n+2)}+\frac{Q^{1.9}-q(0.6)^{1.9}}{400(1.9)}
$$

Using (3.9), the downtime phase integral for the third holding-cost interval is expressed as:

$$
\int_{0.6}^{T} q(t) d t=\frac{q(0.6)^{1.9}-L^{1.9}}{400(1.9)} .
$$

Finally, (3.8) is used to develop boundary-value constraints for $q(t)$ at interval border point $\tau_{1}=0.6$ in the downtime phase.

$$
q(0.6)=\left[Q^{0.9}-400(0.9)\left(0.6-t_{1}\right)\right]^{\frac{1}{0.9}} .
$$

Step 3b-5b:

Varying the values of $L$, and going through the required steps, the best solution for the current holding-cost pair of intervals $\left(t_{1}\right.$ in the second interval and $T$ in the third) is given below:

$$
\begin{aligned}
& L=310, q(0.3)=394.318, Q=448, t_{1}=0.500286, T=0.69093, S=500 \\
& \pi_{i, j}(Q, L)=19030.97 .
\end{aligned}
$$

Step 6b:

Since this solution has a higher profit $\pi_{i, j}(Q, L)$ than the one obtained in step $5 \mathrm{a}$, we proceed to the next pair of intervals for $t_{1}$ and $T$ by moving $T$ from the third to the second holding-cost interval

(c). Both $t_{1}$ and $T$ are in the second interval.

Step 2c:

Assuming both $t_{1}$ and $T$ are in the second holding-cost interval $\left(0.3 \leq t_{1} \leq\right.$ 0.6 , and $0.3 \leq T \leq 0.6)$, then the lot size is in the second purchase-cost range $(300 \leq S<500)$. Since $t_{1}<T \leq 0.6$, then $S=P t_{1}<500$. To formulate the NLP model, the objective function (5.4) is modified as follows:

Maximize

$$
\pi_{i, j}(Q, L)=\frac{1}{T}\left[\begin{array}{l}
1000(70) t_{1}-1000(40) t_{1}-300 \\
-40\left(0.12 \int_{0}^{0.3} q(t) d t+0.16 \int_{0.3}^{T} q(t) d t+\right)
\end{array}\right] .
$$

The objective (5.8) is maximized subject to constraints (4.8), (4.9), (5.5), and (5.6), in addition to the following constraint. The constraint on the integral for 
the second interval, overlapping the uptime and downtime phases, is formulated from (5.2) as follows:

$$
\int_{0.3}^{T} q(t) d t=\sum_{n=0}^{\infty} \frac{0.4^{n}\left[Q^{0.1 n+2}-q(0.3)^{0.1 n+2}\right]}{1000(0.1 n+2)}+\frac{Q^{1.9}-L^{1.9}}{400(1.9)}
$$

Step 3c-5c:

Proceeding with the algorithm steps for all feasible values of $L$, the best solution when both $t_{1}$ and $T$ are in the second interval is given below:

$L=223, q(0.3)=313.146, Q=348, t_{1}=0.4220637, T=0.599759, S=422$, $\pi_{i, j}(Q, L)=15274.59$.

Step 6:

Since this solution leads to a lower profit than the one obtained in step 5b, then this is the last step of the algorithm. The best solution of the previous holding-cost interval pair ( $t_{1}$ in the second interval and $T$ in the third interval), is identified as the final optimum solution. This solution, found in step $5 \mathrm{~b}$, is summarized below:

$$
L=310, \quad Q=448, \quad t_{1}=0.5, \quad T=0.69, \quad \pi_{i, j}(Q, L)=19030.97 .
$$

The solution of the above example using the model proposed by Alfares [2] is given by $\left(L=0, Q=126, t_{1}=0.312, T=0.528, S=312\right)$. Keeping the original no-discount feature of the Alfares [2] model, and considering the non-discounted unit purchase price $\left(c_{1}=50\right)$, gives a profit of $\pi_{i, j}(Q, L)=10783.01$. If we consider the discounted purchase price $\left(c_{2}=45\right)$ corresponding to $S=312$, then the profit per unit time increases to $\pi_{i, j}(Q, L)=13774.35$. Comparing 13774.35 to 19030.97 , the current model increases the profit per period by $38 \%$, even if discounts are added to the model of Alfares [2]. Therefore, it can be concluded that the current model produces significantly higher profits than the model of Alfares [2].

\subsection{Sensitivity analysis for incremental holding cost}

Sensitivity analysis was conducted for the retroactive holding cost example, by individually changing each input parameter by steps of $-20 \%,-10 \%,+10 \%$, and $+20 \%$. Since four changes were applied to six different parameters $(D, P$, $\left.K, \delta, h_{i}, c_{j}\right), 24$ additional problems were solved. The results are summarized in Table 4. As expected, the results are very similar to the retroactive holding cost case. The total profit per unit time $\pi_{i, j}(Q, L)$ is positively correlated with the demand rate $D$ and the selling price $\delta$, and negatively correlated with all the cost parameters $\left(K, h_{i}, c_{j}\right)$. For the production rate $P$, however, the profit shows mixed behavior. Interestingly, the relative changes in the objective functions are almost identical for the two cases. 
Table 4. Sensitivity analysis for the incremental holding cost example.

\begin{tabular}{|c|c|c|c|c|c|c|c|c|}
\hline Parameter & Original value & New values & $L$ & $Q$ & $t_{1}$ & $T$ & $S$ & $\pi_{i, j}(Q, L)$ \\
\hline \multirow[b]{4}{*}{$D$} & & 320 & 175 & 394 & 0.500 & 0.890 & 500 & 14692.81 \\
\hline & & 360 & 228 & 408 & 0.500 & 0.782 & 500 & 16841.59 \\
\hline & & 440 & 335 & 455 & 0.600 & 0.750 & 600 & 21172.66 \\
\hline & 400 & 480 & 460 & 518 & 0.535 & 0.600 & 535 & 23497.80 \\
\hline \multirow[b]{4}{*}{$P$} & & 800 & 341 & 390 & 0.626 & 0.694 & 500 & 19041.16 \\
\hline & & 900 & 315 & 422 & 0.601 & 0.750 & 541 & 19003.84 \\
\hline & & 1100 & 265 & 455 & 0.500 & 0.764 & 550 & 18990.22 \\
\hline & 1000 & 1200 & 243 & 483 & 0.500 & 0.834 & 600 & 18952.44 \\
\hline \multirow[b]{4}{*}{ K } & & 240 & 310 & 448 & 0.500 & 0.691 & 500 & 19117.81 \\
\hline & & 270 & 310 & 448 & 0.500 & 0.691 & 500 & 19074.39 \\
\hline & & 330 & 310 & 448 & 0.500 & 0.691 & 500 & 18987.55 \\
\hline & 300 & 360 & 310 & 448 & 0.500 & 0.691 & 500 & 18944.13 \\
\hline \multirow[b]{4}{*}{$\delta$} & & 56 & 107 & 270 & 0.501 & 0.743 & 500 & 9219.53 \\
\hline & & 63 & 205 & 354 & 0.500 & 0.713 & 500 & 14039.26 \\
\hline & & 77 & 420 & 549 & 0.500 & 0.674 & 500 & 24160.57 \\
\hline & 70 & 84 & 523 & 645 & 0.501 & 0.662 & 500 & 29405.71 \\
\hline \multirow[b]{4}{*}{$h_{1}, h_{2}, h_{3}$} & & $0.10,0.13,0.16$ & 408 & 538 & 0.501 & 0.676 & 500 & 19485.60 \\
\hline & & $0.11,0.145,0.18$ & $\begin{array}{l}408 \\
355\end{array}$ & $\begin{array}{l}530 \\
489\end{array}$ & $\begin{array}{l}0.501 \\
0.500\end{array}$ & 0.683 & 500 & 19246.60 \\
\hline & & $0.13,0.175,0.22$ & 270 & 412 & 0.501 & 0.699 & 500 & 18834.77 \\
\hline & $0.12,0.16,0.20$ & $0.14,0.19,0.24$ & 240 & 385 & 0.500 & 0.705 & 500 & 18655.80 \\
\hline \multirow[b]{4}{*}{$c_{1}, c_{2}, c_{3}$} & & $40,36,32$ & & & & & & \\
\hline & & $45,40.5,36$ & $\begin{array}{l}589 \\
419\end{array}$ & 548 & $\begin{array}{l}0.500 \\
0.500\end{array}$ & $\begin{array}{l}0.655 \\
0.674\end{array}$ & $\begin{array}{l}500 \\
500\end{array}$ & $\begin{array}{l}25561.68 \\
22219.80\end{array}$ \\
\hline & & $55,49.5,44$ & 214 & 362 & 0.500 & 0.711 & 500 & 15977.31 \\
\hline & $50,45,40$ & $60,54,48$ & 144 & 301 & 0.500 & 0.729 & 500 & 13047.11 \\
\hline
\end{tabular}

\section{Conclusions}

This paper presented mathematical models and solution procedures for a production planning system with several realistic features. The main characteristics of the system are: customer demand is a function of the current inventory level, unit inventory holding cost is a function of the unit purchase cost and the storage duration, unit purchase cost is a function of the order size, and a new lot is produced gradually at a finite rate. Since the demand is not constant, the sales revenue is not fixed. Therefore, the objective of the system is to maximize the net profit instead of minimizing the total cost. In the proposed system, higher inventory levels generate greater demands and therefore increase revenues, but at the expense of incurring higher holding costs. Therefore, the initial and ending inventory level is allowed to be a non-zero decision variable whose value is optimally determined by the model.

Mathematical models have been developed for two types of holding-cost increase with longer storage duration: retroactive and incremental. For the retroactive holding cost case, nonlinear programming is used to find the optimum solution. For the incremental holding cost case, a direct solution of the NLP model is too difficult. Therefore, an efficient optimum search procedure is developed. Real-life decision makers can use these techniques to increase profits, by utilizing quantity discounts to reduce purchasing costs and maintaining higher inventory levels to increase the demand, without excessively increasing 
the holding costs.

This work presented in this paper can be extended in several directions. One direction is to explore alternative functional forms of the holding cost, purchase cost, and customer demand variability. For example, the unit holding cost could be divided into two components: an opportunity cost proportional to the purchase cost, and a fixed cost that includes all other carrying costs. Another direction is to consider other factors such as the sale price and the production rate as decision variables. Finally, extending the model to consider multiple items would present a very interesting and challenging research problem.

\section{Acknowledgment}

The author would like to express gratitude for the support and research facilities provided by King Fahd University of Petroleum \& Minerals (KFUPM).

\section{References}

[1] H.K. Alfares. Inventory model with stock-level dependent demand rate and variable holding cost. International Journal of Production Economics, 108(12):259-265, 2007. http://dx.doi.org//10.1016/j.ijpe.2006.12.013.

[2] H.K. Alfares. Production-inventory system with finite production rate, stockdependent demand, and variable holding cost. RAIRO-Operations Research, 48(1):135-150, 2014. http://dx.doi.org/10.1051/ro/2013058.

[3] W.C. Benton and S. Park. A classification of literature on determining the lot size under quantity discounts. European Journal of Operational Research, 92(2):219-238, 1996. http://dx.doi.org/10.1016/0377-2217(95)00315-0.

[4] D. Das, A. Roy and S. Kar. Improving production policy for a deteriorating item under permissible delay in payments with stock-dependent demand rate. Computers and Mathematics with Applications, 60(7):1973-1985, 2010. http://dx.doi.org/10.1016/j.camwa.2010.07.031.

[5] Y. Duan, G. Li, J.M. Tien and J. Huo. Inventory models for perishable items with inventory level dependent demand rate. Applied Mathematical Modelling, 36(10):5015-5028, 2012. http://dx.doi.org/10.1016/j.apm.2011.12.039.

[6] V. Gupta and S.R. Singh. An integrated inventory model with fuzzy variables, three-parameter Weibull deterioration and variable holding cost under inflation. Int. J. of Operational Research, 18(4):434-451, 2013. http://dx.doi.org/10.1504/IJOR.2013.057485.

[7] T.P. Hsieh and Ch.Y. Dye. Optimal replenishment policy for perishable items with stock-dependent selling rate and capacity constraint. Computers and Industrial Engineering, 59(2):251-258, 2010. http://dx.doi.org/10.1016/j.cie.2010.04.006.

[8] J. Hu and C.L. Munson. Dynamic demand lot-sizing rules for incremental quantity discounts. Journal of the Operational Research Society, 53(8):855-863, 2002. http://dx.doi.org/10.1057/palgrave.jors.2601373.

[9] J. Hu, C.L. Munson and E.A. Silver. A modified Silver-Meal heuristic for dynamic lot sizing under incremental quantity discounts. Journal of the Operational Research Society, 55(6):671-673, 2004. http://dx.doi.org/10.1057/palgrave.jors.2601679. 
[10] T.Y. Lin. An economic order quantity with imperfect quality and quantity discounts. Applied Mathematical Modelling, 34(10):3158-3165, 2010. http://dx.doi.org/10.1016/j.apm.2010.02.004.

[11] A. Mendoza and J.A. Ventura. Incorporating quantity discounts to the $\{$ EOQ model with transportation costs. International Journal of Production Economics, 113(2):754-765, 2008. http://dx.doi.org/10.1016/j.ijpe.2007.10.010.

[12] V.K. Mishra and L. Sahab Singh. Deteriorating inventory model for time dependent demand and holding cost with partial backlogging. International Journal of Management Science and Engineering Management, 6(4):267-271, 2011. http://dx.doi.org/10.1080/17509653.2011.10671172.

[13] C.L. Munson and M.J. Rosenblatt. Theories and realities of quantity discounts: An exploratory study. Production and Operations Management, 7(4):352-369, 1998. http://dx.doi.org/10.1111/j.1937-5956.1998.tb00129.x.

[14] V. Pando, J. García-Laguna and L.A. San-José. Optimal policy for profit maximising in an EOQ model under non-linear holding cost and stock-dependent demand rate. International Journal of Systems Science, 43(11):2160-2171, 2012. http://dx.doi.org/10.1080/00207721.2011.565134.

[15] V. Pando, J. García-Laguna, L.A. San-José and J. Sicilia. Maximizing profits in an inventory model with both demand rate and holding cost per unit time dependent on the stock level. Computers and Industrial Engineering, 62(2):599608, 2012. http://dx.doi.org/10.1016/j.cie.2011.11.009.

[16] V. Pando, L.A. San-José, J. García-Laguna and J. Sicilia. An economic lot-size model with non-linear holding cost hinging on time and quantity. International Journal of Production Economics, 145(1):294-303, 2013. http://dx.doi.org/10.1016/j.ijpe.2013.04.050.

[17] A. Roy. An inventory model for deteriorating items with price dependent demand and time-varying holding cost. Advanced Modeling and Optimization, 10(1):2537, 2008.

[18] A. Roy, M.K. Maiti, S. Kar and M. Maiti. An inventory model for a deteriorating item with displayed stock dependent demand under fuzzy inflation and time discounting over a random planning horizon. Applied Mathematical Modelling, 33(2):744-759, 2009. http://dx.doi.org/10.1016/j.apm.2007.12.015.

[19] L.A. San-José and J. García-Laguna. Optimal policy for an inventory system with backlogging and all-units discounts: Application to the composite lot size model. European Journal of Operational Research, 192(3):808-823, 2009. http://dx.doi.org/10.1016/j.ejor.2007.10.026.

[20] B. Sarkar. An $\{E O Q\}$ model with delay in payments and stock dependent demand in the presence of imperfect production. Applied Mathematics and Computation, 218(17):8295-8308, 2012. http://dx.doi.org/10.1016/j.amc.2012.01.053.

[21] Z. Sazvar, A. Baboli and M.R.A. Jokar. A replenishment policy for perishable products with non-linear holding cost under stochastic supply lead time. The International Journal of Advanced Manufacturing Technology, 64(5-8):1087-1098, 2013. http://dx.doi.org/10.1007/s00170-012-4042-2.

[22] N.H. Shah, H.N. Soni and K.A. Patel. Optimizing inventory and marketing policy for non-instantaneous deteriorating items with generalized type deterioration and holding cost rates. Omega, 41(2):421-430, 2013. http://dx.doi.org/10.1016/j.omega.2012.03.002. 
[23] J.T. Teng and C.T. Chang. Economic production quantity models for deteriorating items with price- and stock-dependent demand. Computers and Operations Research, 32(2):297-308, 2005. http://dx.doi.org/10.1016/S03050548(03)00237-5.

[24] T.L. Urban. An extension of inventory models with discretely variable holding costs. International Journal of Production Economics, 114(1):399-403, 2008. http://dx.doi.org/10.1016/j.ijpe.2008.02.014.

[25] M.I.M. Wahab and M.Y. Jaber. Economic order quantity model for items with imperfect quality, different holding costs, and learning effects: A note. Computers and Industrial Engineering, 58(1):186-190, 2010. http://dx.doi.org/10.1016/j.cie.2009.07.007.

[26] S. Yang, K.S. Hong and Ch. Lee. Supply chain coordination with stock-dependent demand rate and credit incentives. International Journal of Production Economics, 157(1):105-111, 2014. http://dx.doi.org/10.1016/j.ijpe.2013.06.014.

[27] Q. Zhang, J. Luo and Y. Duan. Buyer-vendor coordination for fixed lifetime product with quantity discount under finite production rate. International Journal of Systems Science, pp. 1-14, 2014. http://dx.doi.org/10.1080/00207721.2014.906684. 